Bull. Austral. Math. Soc.

$46 \mathrm{~A} 25,46 \mathrm{~B} 10$

Vol. $62(2000) \quad[235-241]$

\title{
A CONE CHARACTERISATION OF REFLEXIVE LOCALLY CONVEX SPACES
}

\author{
JINGHUI QIU
}

In this paper, we find the dual relationship between solidness and the angle property of cones, which is characteristic of reflexivity for locally convex spaces.

\section{INTRODUCTION}

Vector optimisation theory originated from decision-making problems where it is often required that decision making be based on optimising several criteria. A vector optimisation problem is therefore to find all efficient points in some vector partial ordering. Hence one needs to consider the various properties of a convex cone which defines a vector partial order in a Banach space (or more generally, in a locally convex space), such as the angle property, property $(\pi)$, solidness, et cetera (see [1]). When one tries to solve a given vector optimisation problem, some scalarisation processes are closely linked to the properties of the corresponding cones. There has been interesting research on the properties of cones and the relationship between them, for example, see $[1,2,3,4,8]$. Particularly, Han [2] proved the following results.

THEOREM 1.1. [2, Theorem 2.1] Let $\Lambda$ be a closed convex cone in a Banach space $X$. Then $\Lambda$ satisfies the angle property if and only if $\Lambda^{*}$ is a solid cone, that is, int $\Lambda^{*} \neq \emptyset$.

TheOREM 1.2. [2, Theorem 2.4] Let $\Lambda$ be a closed convex cone in a reflexive Banach space $X$. Then $\Lambda$ is solid if and only if $\Lambda^{*}$ satisfies the angle property.

Obviously Theorem 1.2 is a direct consequence of Theorem 1.1, since we have $\Lambda^{* *}=\Lambda$ in a reflexive Banach space $X$. Besides, Han [3] pointed out that in a general Banach space (which need not be reflexive) a closed convex cone $\Lambda$ being solid implies $\Lambda^{*}$ satisfies the angle property. Han [3] conjectured that the converse is also true (even in a locally convex space), that is, $\Lambda^{*}$ satisfying the angle property implies $\Lambda$ is solid. In [6], we introduced the angle property for convex cones in locally convex spaces and generalised Theorem 1.1 as follows.

Received 8th December, 1999

Copyright Clearance Centre, Inc. Serial-fee code: 0004-9727/00 \$A2.00+0.00. 
TheOREM 1.3. [6, Theorem 2.1] Let $\Lambda$ be a closed convex cone in a locally convex space. $X$. Then $\Lambda$ satisfies the angle property if and only if $\Lambda^{*}$ is a solid cone in $\left(X^{*}, \beta\left(X^{*}, X\right)\right)$.

Furthermore, we [6, Counterexamples] constructed a class of closed convex cones $\Lambda$ in some Banach spaces with Schauder bases, which are not solid but whose polar cones $\Lambda^{*}$ satisfy the angle property. Certainly, those Banach spaces are not reflexive. Thus we negatively answered the conjecture raised by Han. Motivated by the above known results, in this paper we try to find the dual relationship between solidness and the angle property, which is characteristic of reflexivity for locally convex spaces. As a corollary, we shall obtain a cone character of reflexivity for Fréchet spaces (particularly, for Banach spaces).

\section{A Cone Characterisation of Reflexivity}

Let $X$ be a real locally convex Hausdorff topological vector space (briefly, denoted by a locally convex space) and let $X^{*}$ be its topological dual. We denote the topologies on $X^{*}$ of uniform convergence on bounded subsets and finite subsets of $X$ by $\beta\left(X^{*}, X\right)$ and $\sigma\left(X^{*}, X\right)$ respectively (for example, see $[5,7,9]$ ). $\left(X^{*}, \beta\left(X^{*}, X\right)\right)$ and $\left(X^{*}, \sigma\left(X^{*}, X\right)\right)$ are called the strong dual and weak dual of $X$ respectively. For a given convex cone $\Lambda$ in $X$, we denote the polar of $\Lambda$ by $\Lambda^{*}$; that is, $\Lambda^{*}=\left\{f \in X^{*}: f(x) \leqslant 0\right.$ for all $\left.x \in \Lambda\right\}$. Obviously, $\Lambda^{*}$ is also a convex cone, and it is called the polar cone of $\Lambda$ in $X^{*}$. If int $\Lambda \neq \emptyset$, then $\Lambda$ is said to be solid. If $\Lambda \cap(-\Lambda)=\{0\}$, then $\Lambda$ is said to be proper. If there exists a closed convex set $A$ in $X$ such that $0 \notin A$ and $\Lambda=\Lambda(A):=\{t x: t \geqslant 0, x \in A\}$, then $A$ is said to be a base of $\Lambda$. Moreover, if $A$ is bounded, then $\Lambda$ is said to admit a bounded base. In a Banach space, a closed convex cone $\Lambda$ is said to have the angle property if there exists $f \in X^{*} \backslash\{0\}$ and $0<\varepsilon \leqslant 1$ such that $\Lambda \subset\{x \in X: f(x) \leqslant-\varepsilon\|f\|\|x\|\}$ (see [1] or [2]). In fact, the angle property can be restated as follows: there exists $f \in X^{*} \backslash\{0\}$ and $\alpha>0$ such that $\Lambda \subset\{x \in X: f(x) \leqslant-\alpha\|x\|\}$. In [6], we extended the concept of angle properties to closed convex cones in locally convex spaces as follows.

Definition 2.1: A closed convex cone $\Lambda$ in a locally convex space $X$ is said to have the angle property if there exists $f \in X^{*} \backslash\{0\}$ such that for any member $p$ of a generating family $\mathcal{P}$ of semi-norms on $X$ (see $[7, \mathrm{p} .48]$ ), there is $\alpha_{p}>0$ satisfying $f(x) \leqslant-\alpha_{p} p(x)$ for all $x \in \Lambda$.

We know that a closed convex cone $\Lambda$ satisfies the angle property if and only if it admits a bounded base (for the case of Banach spaces, see [2, Theorem 2.1]; for the case of locally convex spaces, see [6, Theorem 2.1]). Thus for a closed convex cone $\Lambda$ in a locally convex space $X$, the following are equivalent to each other:

(i) $\Lambda$ satisfies the angle property; 
(ii) $\Lambda^{*}$ is solid in $\left(X^{*}, \beta\left(X^{*}, X\right)\right)$;

(iii) $\Lambda$ admits a bounded base.

We should remark that Jameson [4, Theorem 3.8.4] has obtained the equivalence between (ii) and (iii) previously. His following result is also very useful (see [4, p.121]).

Lemma 2.1. Let $\Lambda$ be a convex cone in a locally convex space $X$. If $\Lambda$ admits a bounded base then $\Lambda$ is closed.

Let $(Z, \mathcal{T})$ be a locally convex space, let $Z^{*}:=(Z, \mathcal{T})^{*}$ be its topological dual and $Z^{* *}$ be the toplogical dual of $\left(Z^{*}, \beta\left(Z^{*}, Z\right)\right)$, that is, the bidual of $(Z, \mathcal{T})$. If $\Lambda \subset Z^{*}$ is a convex cone, then we denote the polar of $\Lambda$ taken in $Z^{* *}$ by $\Lambda^{*}$ and the polar of $\Lambda$ taken in $Z$ by $\Lambda_{*}$; that is, $\Lambda^{*}=\left\{l \in Z^{* *}: l(f) \leqslant 0\right.$ for all $\left.f \in \Lambda\right\}$ and $\Lambda_{*}=\{x \in Z: f(x) \leqslant 0$ for all $f \in \Lambda\}$. Briefly, we denote the closure of $\Lambda$ in $\left(Z^{*}, \sigma\left(Z^{*}, Z\right)\right)$ by $\bar{\Lambda}^{\sigma}$. The following Lemma is the key to the proof of our main result.

Lemma 2.2. Let $\left(Z^{*}, \beta\left(Z^{*}, Z\right)\right)$ be the strong dual of a locally convex space $(Z, \mathcal{T})$ and let $\Lambda$ be a convex cone in $Z^{*}$. If there exists a closed convex set $A$ in $\left(Z^{*}, \beta\left(Z^{*}, Z\right)\right)$ such that $0 \notin A, 0 \in \bar{A}^{\sigma}$ and $\Lambda=\Lambda(A):=\{t f: t \geqslant 0, f \in A\} ;$ then there is no bounded convex set $B$ in $\left(Z^{*}, \beta\left(Z^{*}, Z\right)\right)$ such that $B$ is $\sigma\left(Z^{*}, Z\right)$-closed, $0 \notin B$ and $\bar{\Lambda}^{\sigma}=\Lambda(B):=\{t f: t \geqslant 0, f \in B\}$.

Proof: Assume the contrary, that there is a bounded convex set $B$ in $\left(Z^{*}, \beta\left(Z^{*}, Z\right)\right)$ such that $B$ is $\sigma\left(Z^{*}, Z\right)$-closed, $0 \notin B$ and $\bar{\Lambda}^{\sigma}=\Lambda(B):=\bigcup_{t \geqslant 0} t B$. Since $B$ is a $\sigma\left(Z^{*}, Z\right)$-closed convex set and $0 \notin B$, by the Hahn-Banach separation theorem there is $x_{0} \in Z, x_{0} \neq 0$ such that $f\left(x_{0}\right) \leqslant-1$ for all $f \in B$. Put $K:=\left(\widehat{x_{0}}=-1\right) \cap \bar{\Lambda}^{\sigma}$, where $\left(\widehat{x_{0}}=-1\right)$ denotes the set $\left\{f \in Z^{*}: f\left(x_{0}\right)=-1\right\}$. Then for any $f \in K \subset \bar{\Lambda}^{\sigma} \backslash\{0\} \subset \bigcup_{t>0} t B$, there is $\mu>0$ such that $f \in \mu B$, or $f / \mu \in B$. Thus $f\left(x_{0}\right) / \mu \leqslant-1$, or $f\left(x_{0}\right) \leqslant-\mu$. Since $f \in K \subset\left(\widehat{x_{0}}=-1\right), f\left(x_{0}\right)=-1$. Hence $-1 \leqslant-\mu$, that is, $\mu \leqslant 1$. Thus $f \in \mu B \subset[0,1] B$ and $K \subset[0,1] B$ is $\beta\left(Z^{*}, Z\right)$ bounded. Here $[0,1] B$ denotes the set $\{t b: 0 \leqslant t \leqslant 1, b \in B\}$. Since $\left(\widehat{x_{0}}=-1\right)$ and $\bar{\Lambda}^{\sigma}$ are both $\sigma\left(Z^{*}, Z\right)$-closed, $K=\left(\widehat{x_{0}}=-1\right) \cap \bar{\Lambda}^{\sigma}$ is $\sigma\left(Z^{*}, Z\right)$-closed, and clearly $0 \notin K$. Next we show that $\bar{\Lambda}^{\sigma}=\Lambda(K)$. Obviously, $K \subset[0,1] B \subset \bigcup_{t \geqslant 0} t B=\bar{\Lambda}^{\sigma}$. On the other hand, by the assumption that $\bar{\Lambda}^{\sigma}=\bigcup_{t \geqslant 0} t B$, for any $f \in \bar{\Lambda}^{\sigma}$ with $f \neq 0$, there is $\lambda>0$ and $b \in B$ such that $f=\lambda b$, or $f / \lambda=b \in B$. Hence $f\left(x_{0}\right) / \lambda \leqslant-1$ and $f\left(x_{0}\right) \leqslant-\lambda<0$. Clearly,

$$
-\left(1 / f\left(x_{0}\right)\right)>0 \text { and }-\left(1 / f\left(x_{0}\right)\right) f \in\left(\widehat{x_{0}}=-1\right) \cap \bar{\Lambda}^{\sigma}=K .
$$

Therefore $\bar{\Lambda}^{\sigma}=\Lambda(K)$. For any $f \in \bar{\Lambda}^{\sigma} \backslash\{0\}$, put $\lambda_{f}:=-1 / f\left(x_{0}\right)$, then $\lambda_{f}>0$ and $\lambda_{f} f \in K$. By the hypothesis, $A \subset \Lambda \subset \bar{\Lambda}^{\sigma}$ and $0 \notin A$. We consider the set 
$\left\{\lambda_{f}: f \in A\right\}$ of positive real numbers. Assume that there is a sequence $\left\{f_{n}\right\}_{n \in N} \subset A$ such that $\lambda_{f_{n}} \stackrel{n}{\longrightarrow} \infty$. Since $0 \notin A$ and $A$ is $\beta\left(Z^{*}, Z\right)$-closed, there is an absolutely convex 0-neighbourhood $V$ in $\left(Z^{*}, \beta\left(Z^{*}, Z\right)\right)$ such that $V \cap A=\emptyset$. For every $n \in N$, $f_{n} \in A$, hence $f_{n} \notin V$ and $\lambda_{f_{n}} f_{n} \notin \lambda_{f_{n}} V$. By noting that every $\lambda_{f_{n}} f_{n} \in K$, we have $K \not \subset \lambda_{f_{n}} V$ for every $n \in N$. This contradicts the previous conclusion that $K$ is $\beta\left(Z^{*}, Z\right)$-bounded. Thus we have shown that $\left\{\lambda_{f}: f \in A\right\}$ is bounded, that is, there exists $\beta>0$ such that $\lambda_{f} \leqslant \beta$ for all $f \in A$. Since $0 \notin K$ and $K$ is $\sigma\left(Z^{*}, Z\right)$ closed, there exists an absolutely convex 0 -neighbourhood $W$ in $\left(Z^{*}, \sigma\left(Z^{*}, Z\right)\right)$ such that $W \cap K=\emptyset$. Obviously, $(1 / \beta) W$ is still a 0 -neighbourhood in $\left(Z^{*}, \sigma\left(Z^{*}, Z\right)\right)$. Since $0 \in \bar{A}^{\sigma}$, we have that $(1 / \beta) W \cap A \neq \emptyset$, that is, there is $f_{0} \in A$ such that $f_{0} \in(1 / \beta) W$. Since $W$ is absolutely convex and $0<\lambda_{f_{0}} \leqslant \beta$, we have:

$$
f_{0} \in \frac{1}{\beta} W \subset \frac{1}{\lambda_{f_{0}}} W, \text { or } \quad \lambda_{f_{0}} f_{0} \in W
$$

On the other hand, $\lambda_{f_{0}} f_{0} \in K$ and hence $\lambda_{f_{0}} f_{0} \in W \cap K$, which contradicts the assumption that $W \cap K=\emptyset$. Thus we have completed the proof of Lemma 2.2.

LEMMA 2.3. Let $(Z, \mathcal{T})$ be a complete locally convex space which is not semireflexive. Then there exists a bounded closed convex set $A$ in $\left(Z^{*}, \beta\left(Z^{*}, Z\right)\right)$ which is not closed in $\left(Z^{*}, \sigma\left(Z^{*}, Z\right)\right)$.

Proof: Let $Z^{* *}$ denote the bidual of $(Z, \mathcal{T})$. Since $(Z, \mathcal{T})$ is not semi-reflexive, there exists $l \in Z^{* *} \backslash Z$. Put $H:=\left\{f \in Z^{*}: l(f)=1\right\}$, then $H$ is a closed hyperplane in $\left(Z^{*}, \beta\left(Z^{*}, Z\right)\right)$. Let $\mathcal{U}$ be a base of 0 -neighbourhoods in $(Z, \mathcal{T})$. We assert that there is $U \in \mathcal{U}$ such that $H \cap U^{\circ}$ is not $\sigma\left(Z^{*}, Z\right)$-closed. If not, $H$ would be an $a w^{*}$-closed hyperplane. Since $(Z, \mathcal{T})$ is complete, $H$ must be $\sigma\left(Z^{*}, Z\right)$-closed (see $[7$, p.149] or [9, Corollary 12-2-16]), which contradicts that $l \in Z^{* *} \backslash Z$. Put $A:=H \cap U^{\circ}$, then $A$ is convex and $\beta\left(Z^{*}, Z\right)$-closed. Since $A \subset U^{\circ}$ and $U^{\circ}$ is $\beta\left(Z^{*}, Z\right)$-bounded, $A$ is $\beta\left(Z^{*}, Z\right)$-bounded. In a word, $A$ is a bounded closed convex set in $\left(Z^{*}, \beta\left(Z^{*}, Z\right)\right)$, but it is not $\sigma\left(Z^{*}, Z\right)$-closed.

THEOREM 2.1. Let $(Z, \mathcal{T})$ be a complete locally convex space. If $(Z, \mathcal{T})$ is not semi-reflexive, then there exists a closed convex cone $\Lambda$ satisfying the angle property (or equivalently, admitting a bounded base) in $\left(Z^{*}, \beta\left(Z^{*}, Z\right)\right.$ ) whose polar $\Lambda_{*}$ is not solid in $(Z, \mathcal{T})$.

Proof: By Lemma 2.3, we know that there exists a bounded closed convex set $A$ in $\left(Z^{*}, \beta\left(Z^{*}, Z\right)\right)$ which is not closed in $\left(Z^{*}, \sigma\left(Z^{*}, Z\right)\right)$. Hence there is $f_{0} \in \bar{A}^{\sigma}$, with $f_{0} \notin A$. Without loss of generality, we may assume that $f_{0}=0$. Put $\Lambda=\Lambda(A):=$ $\{t f: t \geqslant 0, f \in A\}$. Then by Lemma $2.1, \Lambda$ is a closed convex cone admitting a bounded base $A$ in $\left(Z^{*}, \beta\left(Z^{*}, Z\right)\right)$. We shall prove that $\Lambda_{*}$ is not solid in $(Z, \mathcal{T})$. 
Assume the contrary. There is $x_{0} \in \Lambda_{*}$ and an absolutely convex 0 -neighbourhood $U$ in $(Z, \mathcal{T})$ such that

$$
x_{0}+U \subset \Lambda_{*}:=\{x \in Z: f(x) \leqslant 0 \text { for all } f \in \Lambda\} \text {, that is, } U \subset \Lambda_{*}-x_{0} .
$$

Since $U$ is absolutely convex,

$$
\begin{aligned}
U^{\circ}: & =\left\{f \in Z^{*}:|f(x)| \leqslant 1 \text { for all } x \in U\right\} \\
& =\left\{f \in Z^{*}: f(x) \leqslant 1 \text { for all } x \in U\right\}=: U^{r} \supset\left(\Lambda_{*}-x_{0}\right)^{r} .
\end{aligned}
$$

Put $B:=\left(\widehat{x_{0}}=-1\right) \cap\left(\Lambda_{*}-x_{0}\right)^{r}$, then $B$ is a $\sigma\left(Z^{*}, Z\right)$-closed convex set. It is easy to see that $x_{0} \neq 0$. If not, we would have $U \subset \Lambda_{*}$, which implies that $\Lambda_{*}=Z$ and $\Lambda=\{0\}$. This leads to $A=\{0\}$, which contradicts the assumption that $0 \notin A$. Sjnce $B$ is $\sigma\left(Z^{*}, Z\right)$-closed, $U^{\circ}$ is $\sigma\left(Z^{*}, Z\right)$-compact and $B \subset\left(\Lambda_{*}-x_{0}\right)^{r} \subset U^{\circ}$, we conclude that $B$ is $\sigma\left(Z^{*}, Z\right)$-compact. By noting that every $\sigma\left(Z^{*}, Z\right)$-compact convex set is $\beta\left(Z^{*}, Z\right)$-bounded, we know that $B$ is a bounded convex set in $\left(Z^{*}, \beta\left(Z^{*}, Z\right)\right)$. Also, by the definition of $B, B$ is $\sigma\left(Z^{*}, Z\right)$-closed and $0 \notin B$. Next we show that $\bar{\Lambda}^{\sigma}=\Lambda(B)$. For any $g \in B:=\left(\widehat{x_{0}}=-1\right) \cap\left(\Lambda_{*}-x_{0}\right)^{r}$, we have $g\left(x_{0}\right)=-1$ and $g\left(x-x_{0}\right) \leqslant 1$ for all $x \in \Lambda_{*}$. From this,

$$
g(x) \leqslant 1+g\left(x_{0}\right)=1-1=0 \text { for all } x \in \Lambda_{*} .
$$

This means that $g \in\left(\Lambda_{*}\right)^{*}=\bar{\Lambda}^{\sigma}$. Thus we have proved that $B \subset \bar{\Lambda}^{\sigma}$. On the other hand, for any $f \in \bar{\Lambda}^{\sigma}=\left(\Lambda_{*}\right)^{*}$, if $f\left(x_{0}\right)=0$, then $f(x)=f\left(x+x_{0}\right) \leqslant 0$ for all $x \in U$, since $x_{0}+U \subset \Lambda_{*}$. This implies that $f(x)=0$ for all $x \in U$, that is, $f=0$. Thus for any $f \in \bar{\Lambda}^{\sigma} \backslash\{0\}$, we have $f\left(x_{0}\right) \neq 0$ and hence $f\left(x_{0}\right)<0$. Put

$$
g:=-\frac{1}{f\left(x_{0}\right)} f, \text { then } g\left(x_{0}\right)=-1 \text { or } g \in\left(\widehat{x_{0}}=-1\right) .
$$

Also, for any $x \in \Lambda_{*}$,

$$
g\left(x-x_{0}\right)=-\frac{1}{f\left(x_{0}\right)} f\left(x-x_{0}\right)=-\frac{f(x)}{f\left(x_{0}\right)}+1 \leqslant 1, \text { that is, } g \in\left(\Lambda_{*}-x_{0}\right)^{r} .
$$

Hence $g \in\left(\widehat{x_{0}}=-1\right) \cap\left(\Lambda_{*}-x_{0}\right)^{r}=: B$. Clearly, for any $f \in \bar{\Lambda}^{\sigma} \backslash\{0\}$,

$$
f=-f\left(x_{0}\right)\left(-\frac{1}{f\left(x_{0}\right)} f\right)=-f\left(x_{0}\right) g \in \bigcup_{t>0} t B \subset \Lambda(B) .
$$

Thus we have shown that $\bar{\Lambda}^{\sigma}=\Lambda(B)$. Now on the one hand, $\Lambda=\Lambda(A)$, where $A$ is a closed convex set in $\left(Z^{*}, \beta\left(Z^{*}, Z\right)\right), 0 \notin A$ and $0 \in \bar{A}^{\sigma}$. On the other hand, 
$\bar{\Lambda}^{\sigma}=\Lambda(B)$, where $B$ is a bounded convex set in $\left(Z^{*}, \beta\left(Z^{*}, Z\right)\right), B$ is $\sigma\left(Z^{*}, Z\right)$-closed and $0 \notin B$. However, this is impossible by Lemma 2.2. This shows that $\Lambda_{*}$ is not solid in $(Z, \mathcal{T})$.

THEOREM 2.2. Let $(Z, \mathcal{T})$ be a complete barrelled space (or equivalently, a complete quasi-barrelled space, see [5, p.368]). Then $(Z, \mathcal{T})$ is reflexive if and only if for any closed convex cone satisfying the angle property in $\left(Z^{*}, \beta\left(Z^{*}, Z\right)\right), \Lambda_{*}$ is solid in $(Z, \mathcal{T})$.

\section{PROOF:}

(i) Assume that $(Z, \mathcal{T})$ is not reflexive. Since $(Z, \mathcal{T})$ is barrelled, $(Z, \mathcal{T})$ is not semi-reflexive. By Theorem 2.1, there exists a closed convex cone $\Lambda$ satisfying the angle property in $\left(Z^{*}, \beta\left(Z^{*}, Z\right)\right)$ such that $\Lambda_{*}$ is not solid in $(Z, \mathcal{T})$.

(ii) Assume that $(Z, \mathcal{T})$ is reflexive, that is, $(Z, \mathcal{T})=\left(Z, \beta\left(Z, Z^{*}\right)\right)$ and $Z^{* *}=Z$. Then for any closed convex cone $\Lambda$ satisfying the angle property in $\left(Z^{*}, \beta\left(Z^{*}, Z\right)\right)$, by Theorem $1.3, \Lambda^{*}$ is a solid cone in $\left(Z^{* *}, \beta\left(Z^{* *}, Z^{*}\right)\right)$. Since $\Lambda_{*}=\Lambda^{*}$ and $(Z, \mathcal{T})=\left(Z, \beta\left(Z, Z^{*}\right)\right)=$ $\left(Z^{* *}, \beta\left(Z^{* *}, Z^{*}\right)\right)$, we know that $\Lambda_{*}$ is solid in $(Z, \mathcal{T})$.

Corollary 2.1. Let $(Z, d)$ be a Fréchet space (that is, a complete metrisable locally convex space). Then $(Z, d)$ is reflexive if and only if for each closed convex cone $\Lambda$ with the angle property in $\left(Z^{*}, \beta\left(Z^{*}, Z\right)\right), \Lambda_{*}$ is a solid cone in $(Z, d)$.

Corollary 2.2. Let $(Z,\|\|)$ be a Banach space. Then $(Z,\|\|)$ is reflexive if and only if for each closed convex cone $\Lambda$ with the angle property in $\left(Z^{*},\|\|^{*}\right), \Lambda_{*}$ is a solid cone in $(Z,\|\|)$. Here $Z^{*}$ is the topological dual of $(Z,\|\|)$ and for any $f \in Z^{*}$, $\|f\|^{*}:=\sup \{|f(x)|:\|x\| \leqslant 1, x \in Z\}$.

\section{REFERENCES}

[1] L. Cesari and M.B. Suryanarayana, 'Existence theorems for Pareto optimisation: multivalued and Banach space valued functionals', Trans. Amer. Math. Soc. 244 (1978), 37-65.

[2] Z.Q. Han, 'Remarks on the angle property and solid cones', J. Optim. Theory Appl. 82 (1994), 149-157.

[3] Z.Q. Han, 'Relations between solid cones and cones satisfying angle property', J. Systems Sci. Math. Sci. 18 (1998), 18-22.

[4] G. Jameson, Ordered linear spaces (Springer-Verlag, Berlin, Heidelberg, New York, 1970).

[5] G. Köthe, Topological vector spaces. I (Springer-Verlag, Berlin, Heidelberg, New York, 1983).

[6] J.H. Qiu, 'On solidness of polar cones' (to appear). 
[7] H.H. Schaefer, Topological vector spaces (Springer-Verlag, Berlin, Heidelberg, New York, 1971).

[8] A. Sterna-Karwat, 'Remarks on convex cones', J. Optim. Theory Appl. 59 (1988), 335-340.

[9] A. Wilansky, Modern methods in topological vector spaces (McGraw-Hill, New York, 1978).

Department of Mathematics

Suzhou University

Suzhou, Jiangsu 215006

People's Republic of China 